\title{
Outgrowing the Immaturity Myth: The Cost of Defending From Neonatal Infectious Disease
}

\author{
Danny Harbeson ${ }^{1 *}$, Rym Ben-Othman ${ }^{2}$, Nelly Amenyogbe ${ }^{1 \dagger}$ and Tobias R. Kollmann ${ }^{1,2 \dagger}$ \\ ${ }^{1}$ Department of Experimental Medicine, University of British Columbia, Vancouver, BC, Canada, ${ }^{2}$ Department of Pediatrics, \\ Division of Infectious Diseases, University of British Columbia, Vancouver, BC, Canada
}

OPEN ACCESS

Edited by:

Ed Clarke,

Medical Research Council The Gambia Unit (MRC), Gambia

Reviewed by:

Scott D. Gray-Owen,

University of Toronto, Canada Adrian John Frederick Luty, Institut de recherche pour le développement (IRD), France

*Correspondence:

Danny Harbeson

dannyharbeson@gmail.com

tThese authors have contributed equally to this work.

Specialty section:

This article was submitted to Vaccines and Molecular Therapeutics,

a section of the journal

Frontiers in Immunology

Received: 07 November 2017 Accepted: 30 April 2018

Published: 29 May 2018

Citation:

Harbeson D, Ben-Othman R, Amenyogbe $N$ and Kollmann TR (2018) Outgrowing the Immaturity Myth: The Cost of Defending From

Neonatal Infectious Disease.

Front. Immunol. 9:1077. doi: 10.3389/fimmu.2018.01077
Newborns suffer high rates of mortality due to infectious disease-this has been generally regarded to be the result of an "immature" immune system with a diminished disease-fighting capacity. However, the immaturity dogma fails to explain (i) greater pro-inflammatory responses than adults in vivo and (ii) the ability of neonates to survive a significantly higher blood pathogen burden than of adults. To reconcile the apparent contradiction of clinical susceptibility to disease and the host immune response findings when contrasting newborn to adult, it will be essential to capture the entirety of available host-defense strategies at the newborn's disposal. Adults focus heavily on the disease resistance approach: pathogen reduction and elimination. Newborn hyperactive innate immunity, sensitivity to immunopathology, and the energetic requirements of growth and development (immune and energy costs), however, preclude them from having an adult-like resistance response. Instead, newborns also may avail themselves of disease tolerance (minimizing immunopathology without reducing pathogen load), as a disease tolerance approach provides a counterbalance to the dangers of a heightened innate immunity and has lower-associated immune costs. Further, disease tolerance allows for the establishment of a commensal bacterial community without mounting an unnecessarily dangerous immune resistance response. Since disease tolerance has its own associated costs (immune suppression leading to unchecked pathogen proliferation), it is the maintenance of homeostasis between disease tolerance and disease resistance that is critical to safe and effective defense against infections in early life. This paradigm is consistent with nearly all of the existing evidence.

Keywords: neonate, infection, defense, tolerance, sepsis

\section{INTRODUCTION}

The world has seen under-five mortality greatly reduced over the last two decades but this progress has least benefited those in the first 28 days of life-the neonatal period-which now accounts for nearly half of all under-five deaths (1). Infectious disease is one of the most common causes of newborn death, accounting for more than a third of all neonatal mortality (1). Unfortunately, the underlying reasons for this are not clear, preventing a rational approach to preventing newborn death across the globe. Unquestionably, newborns are much more susceptible to infection causing clinical disease (2-5). Also clear is that the neonatal immune system is very different than that of adults $(6,7)$. Many immunomodulatory approaches to improving outcome in neonatal infectious disease 
have been unsuccessful $(8,9)$, which necessitates a careful reexamination of our assumptions and beliefs regarding the nature of neonatal immune responses. The current dogmatic view, namely that the neonatal immune system is immature and therefore deficient to resist infection as compared to that of an adult (10), is inadequate as it does not capture the existing body of evidence (11). We recently reviewed the molecular mechanisms guiding the ontogeny of immune response from birth throughout infancy, emphasizing that newborns harbor an immune phenotype that is a match to the unique environmental pressures and challenges in the first days of life $(11,12)$. Balancing disease tolerance and resistance, while a challenge throughout the entire life span, is also unique for newborns (12). We here place the existing evidence in a larger framework to expand on this concept of host defense as a balance between disease tolerance and resistance to help guide the search for actionable answers.

\section{NEONATAL HOST DEFENSE FROM INFECTIOUS DISEASE: THE COMPLETE PICTURE}

Host defense to infection can broadly be divided into three different, not mutually exclusive, categories: disease avoidance, disease resistance, and disease tolerance (13). A more detailed exploration into the finer details of these defense strategies has been previously been outlined by Medzhitov et al. in their 2012 Science paper titled "Disease Tolerance as a Defense Strategy" (13). Here, each strategy is briefly summarized and connected explicitly to the neonatal immune response, which has been found time and time again to be distinct from the adult immune response $(4,11,14-17)$ :

(i) An avoidance strategy reduces the risk of infection by preventing exposure to infectious agents. Human avoidance of rotten meat consumption through an olfactory response to the metabolites produced by bacteria breaking down tissue is an example of avoidance (13). Limitations in both newborn mobility and exclusive breastfeeding can prevent potentially harmful exposure and represent an example of an avoidance strategy in early life (18). However, there are obvious physiological, physical as well as social and cultural limitations to this strategy; there is an unfortunate inevitability to some degree of pathogen exposure.

When avoidance has failed and infection has been established, the (ii) disease resistance approach aims to reduce pathogen burden and has traditionally been considered the primary modus operandi of the immune system (and thus the focus of most prophylactic or therapeutic interventions). However, unleashing antimicrobial immune responses can also cause collateral damage (13). In fact, much of what is clinically recognized as signs and symptoms of infection relates to this immune pathology (19). For example, a recent comparison of sepsis models showed that lipopolysaccharide (LPS) treatment in mice "induced a very similar course of inflammation" as infection (20). Given that LPS has no intrinsic virulence, the pathology of LPS challenge must result from host response, and thus similarities between LPS-induced sepsis and, e.g., polymicrobial sepsis (21) implicate host-mediated immune pathology as a key agent of disease. This is further evidenced by murine studies showing that knocking out anti-inflammatory cytokine production during infection is associated with worse outcomes without impacting bacterial clearance or viral replication $(22,23)$. Importantly, the newborn is particularly susceptible to this host-mediated immune pathology (e.g., intraperitoneal LPS challenge at $10 \mathrm{mg} / \mathrm{kg}$ resulted in $100 \%$ mortality in neonatal mice and $0 \%$ in adults) $(11,24)$. It is therefore not surprising that evolution has selected for a higher threshold that needs to be overcome in early life before a fullfledged immune response can be unleashed (12). This leaves the newborn with a conundrum; a disease avoidance approach has clear limitations [indiscriminately avoiding bacterial colonization is not only impossible, but would be harmful as the first few days of life are extremely important for establishing a healthy and diverse community of commensal enteric bacteria (25)], while a disease resistance approach carries substantial risk for immunemediated damage $(11,13,24,26)$.

Newborns thus likely also rely on employing the third strategy of host defense, disease tolerance. (iii) Disease tolerance reduces potential harm to the host without reducing pathogen burden, generally by minimizing the level of immunopathology that results from a resistance response (13). This strategy is understood to be widely employed by plants (27) but the notion that animals (and humans) may rely on a disease tolerance defense as well has only recently begun to be considered (13). It is important to note that disease tolerance is different from the concept of adaptive immune tolerance: the former is a broad, categorical term for a defense strategy of coping with infection, and the latter is the immunological phenomenon of immune unresponsiveness to specific antigens. To our knowledge, the concept of disease tolerance as a defense strategy in early life has never been experimentally examined. However, existing evidence, while not proof, is at least consistent with its existence. Lastly, the host microbiota has increasingly been recognized as key to host defense, impacting all aspects of from avoidance (colonization resistance) to immune development; however, its role and relation to disease tolerance is significant and unexplored, as the tolerance to a range of microbial commensals is essential for a healthy human host (28).

\section{THE CASE FOR HIGHER DISEASE TOLERANCE IN EARLY LIFE}

A disease tolerant vs. intolerant phenotype would be expected to display a lower morbidity/mortality relative to a same pathogen load, and/or a higher pathogen load at a similar mortality level (13). While many suspected cases of bacterial sepsis in both neonates and adults are not confirmed by a positive blood culture $(29,30)$, within culture-positive cases, neonates have consistently been found to exhibit much higher circulating bacterial loads than adults (31). Despite expected variability depending on the pathogen involved, studies generally report bacterial counts in adults (with an active bacterial infection) to be somewhere in the range of 1-30 CFU/ml blood (31-34), while in neonates, the more commonly detected range lies between 50 and $500 \mathrm{CFU} / \mathrm{ml}$ blood with one-third of infected newborns harboring bacterial counts in excess of $1,000 \mathrm{CFU} / \mathrm{ml}(31,35)$. Furthermore, while $50 \%$ of 
adult culture-positive cases harbor $<1 \mathrm{CFU} / \mathrm{ml}$ blood (considered a "low" bacterial load), 78\% of culture-proven newborn sepsis cases reported $>5 \mathrm{CFU} / \mathrm{ml}$ of blood, and $<50 \mathrm{CFU} / \mathrm{ml}$ blood was considered to be low for neonates (31). Most of these studies were not set up to compare newborn vs. adult bacterial loads in sepsis but rather were framed in the context of describing how much blood would be needed in order to confidently determine a culture-positive or a -negative state, thus do not directly address this comparison (34). However, this relationship also holds true in more controlled animal models, where much higher bacterial counts are consistently found in the blood as well as visceral organs of septic neonatal vs. adult mice challenged with the same pathogen (36).

Perhaps, a higher bacterial load in infected newborns does not seem surprising at first glance-after all, neonates are more susceptible to suffer from infection, and higher bacterial loads would seem to be entirely in line with this observation. However, this simple concept begins to unravel when age-specific mortality statistics are taken into consideration. While bacterial load correlates with outcome across all ages, there are log-fold differences in the scale of circulating bacteria which neonates are able to survive in comparison to adults. Studies have shown $100 \%$ mortality in adult patients with greater than $100 \mathrm{CFU} / \mathrm{ml}$ blood (37) and $84 \%$ mortality when the bacterialload was greater than $5 \mathrm{CFU} / \mathrm{ml}$ blood (38). By contrast, a cohort of neonates with sepsis suffered $73 \%$ mortality when bacterial loads were greater than $1,000 \mathrm{CFU} / \mathrm{ml}$ blood and $37 \%$ when less than 1,000 CFU/ml blood (35). This particular study describes the "low bacterial count with $37 \%$ survival" group as those with bacterial loads between 5 and 49 colonies per $\mathrm{ml}$ of blood-an amount that would be considered extremely high and lethal in adult patients (31). As stated above, the most recent studies tend not to report the magnitude of bacterial burden in human patients with sepsis, but simply whether they were culture positive or negative; this precludes a full assessment of the relationship between bacterial load and mortality across the age groups. However, many animal models using $\mathrm{CFU} / \mathrm{ml}$ blood as an outcome validate the observation that neonates are able to survive much higher circulating bacterial loads than adults $(36,39)$. Note that this is not to suggest that newborns are able to survive higher levels of bacterial exposure than adults (in fact, the opposite is true, as detailed below), rather that neonates are able to survive levels of bacteremia that adults cannot.

\section{THE BALANCE OF DISEASE RESISTANCE VS. IMMUNOPATHOLOGY}

Many studies have described deficiencies in the neonatal innate immune system that could be responsible for the decreased ability to clear invasive pathogens. For example, kinetics of pathogen clearance in animal models of neonatal infection show that neonates take longer to clear invasive bacteria than their adult counterparts $(36,39)$. A recent study comparing methicillinresistant Staphylococcus aureus infection in neonatal and adult mice attributed a delayed clearance in neonates to inefficient phagocytosis and a limited neutrophil recruitment to the site of infection. Specifically, in neonates, neutrophil production dropped off despite the continued presence of bacteria, whereas in adult animals, a diminishing neutrophil production corresponded with bacterial clearance. Other studies have implicated impaired neutrophil recruitment as a potential explanation for the increased susceptibility to infection in early life $(40,41)$. Furthermore, while neonates have higher basal levels of circulating phagocytic cells than adults, they are generally considered to be less efficient phagocytes $(40,42-45)$. For example, in vitro neonatal monocytes and neutrophils in whole blood cultures have been shown to have an impaired phagocytic ability of Escherichia coli and S. aureus when compared to adults (44). However, other groups that found similarly reduced phagocytosis of $S$. aureus by newborn polymorphonuclear leukocytes (PMNs) also found that the exposure of neonatal PMNs to adult plasma resulted in adult levels of bactericidal activity and hydrogen peroxide production (against S. aureus) $(17,46)$. Similarly, phagocytosis of group B Streptococci and E. coli by adult and neonatal purified monocytes had similar phagocytic activity between the different age groups $(45,47)$. This brief excursion into the literature of just one aspect of host defense immediately highlights that the ability of newborn immune cells to fight infection is a purposeful response and not simply a state "deficient as compared to the adult."

Just as in vitro comparisons of neonatal and adult phagocytic cells have contributed to the theory that neonatal susceptibility to infection is a result of "immaturity," so has the evidence accrued which describes diminished in vitro pro-inflammatory responses when comparing neonatal and adult cells $(7,10,48,49)$. However, animal models of neonatal sepsis using a variety of pathogens (both bacterial or viral) or TLR agonists have found neonates to generate an inflammatory response equal to or greater than that of adults $(1,24,39,50-52)$. Furthermore, exogenous supplementations of pro-inflammatory cytokines have been shown to greatly increase mortality in a polymicrobial model of sepsis in neonatal mice $(53,54)$. This increased mortality of neonatal sepsis does not relate to a decreased bacterial clearance, as neonatal mice also suffer a much greater mortality than adults when challenged with purified TLR agonists in the absence of an infection $(24,26)$. This has led to the realization that the inflammatory response itself is considered to be largely responsible for the higher mortality of infected newborns vs. adults $(53,54)$.

Given this higher risk of the newborn vs. adult to suffer from the immune response to an infection (or TLR agonist), newborns would benefit from mechanisms that would reduce the risk to unleash a harmful antimicrobial immune response. The molecular mechanisms related to this have recently begun to be deciphered and highlight a direct connection to disease tolerance. An E. coli model of neonatal sepsis found that neonatal $\mathrm{TRIF}^{-/-}$mice suffered a higher mortality than WT or $\mathrm{MyD} 88^{-/-}$strains with the opposite being true in young adults (55). Neonatal prioritization of TRIF-dependent pathway activation when exposed to TLR agonists was then linked to a strong induction of type 1 interferon regulatory responses, as opposed to the adult MyD88-dependent pro-inflammatory response. A molecular explanation for these age-dependent differences in defense strategy has recently been identified as the endogenous, heterodimeric complex of TLR4 ligands S100A8/A9: high levels of S100A8/A9 shift TLR signaling from MyD88- to TRIF-dependent pathways. S100A8/A9 
alarmins are also known to be massively released at birth. This alarmin release is entirely incongruous with the "immune immaturity" paradigm as it represents a purposeful shift away from MyD88 pathway activation, the preferred adult pathway. If neonatal death was driven by a simple lack of adult-like features, one would expect that any external shift toward a more adult-like immune response would lead to better outcome. But the opposite is in fact the case, as $S 100 a 9^{-/-}$neonatal mice suffer much higher mortality than their WT counterparts when infected, implying the alarmin release at birth; i.e., the subsequent shift away from an adult-like response is an important and necessary step to successfully mount a defense against an early-life infection $(11,56)$. The age-dependent production of S100A8/A9 thus represents an example of disease tolerance unique to neonates that has developed to avoid immunopathology from an MyD88-driven pro-inflammatory response at the potential cost of rapid bacterial clearance.

This emphasis on the TRIF-dependent response is entirely incongruous with the "immune immaturity" paradigm. While this is true for pathogens that signal through TLR4, other mechanisms of disease tolerance to, e.g., Gram-positive infections still need to be identified. For example, there are several other mechanisms in place in early life that commonly are described as immune suppressive, with the notion that these are remnants of the mechanisms that allow semiallogeneic mismatch in utero without rejection of maternal cells by the fetus (12). However, these mechanisms persist far beyond the immediate perinatal period and thus likely have other benefits in postnatal life, such as increasing disease tolerance by reducing immune-mediated pathology ("immune cost") even if it comes at the cost of an increased bacterial burden (13).

\section{THE BALANCE OF DISEASE RESISTANCE VS. DISEASE TOLERANCE}

The benefit of disease tolerance as a host-defense strategy depends on the capacity for virulence of a given invasive agent. If the only pathogen ever encountered by a host organism secreted virulence factors that inflicted mortality in $100 \%$ of cases, there would be intense pressure to improve resistance and no pressure to improve tolerance. More relevant to humans is the opposite case; there are myriads of bacteria that rarely cause mortality and provide both direct and indirect fitness advantages to the host. This creates a situation where disease tolerance is a viable defense strategy, but to a finite degree. Even very low virulence organisms, if left totally unchecked, would cause disease. To prevent disease from occurring upon the transition from the semi-sterile environment in utero into the microbe-rich ex utero world, disease tolerance (immunosuppression preventing immunopathology) and disease resistance (inflammatory/antimicrobial responses preventing virulence) must maintain a state of homeostasis for optimal host defense. Without active suppression of inflammatory innate signaling, the initial influx of microbes from the birth process could prompt an enormous, potentially lethal inflammatory response; even if this inflammatory response did not result in mortality, there would be serious short- and long-term health ramifications as a result of inadequate bacterial diversity in the gut $(25,57)$. If there was no disease resistance, opportunistic colonizers would inevitably reach the blood stream and cause disease (Figure 1). Since adults (a) are less sensitive to immunopathology caused by inflammation, (b) have already established an enteric microbiome, and (c) are not hindered by the energetic requirements of development and environmental change (see below); the benefits of disease resistance (keeping pathogens out) outweigh the costs of disease tolerance (letting pathogens in).

\section{THE COST OF HOST DEFENSE}

Any form of host response (or lack thereof) to an invasive agent must be weighed in terms of the potential for self-inflicted damage, or immunopathology. The resultant immunopathological impact of any given response can range from negligible (i.e., mild fever) to fatal (i.e., septic shock), and thus the immunopathology associated with an immune response has been described as the "immune cost" of a response (58). The three principle host-defense strategies of avoidance, disease tolerance, and disease resistance can be ordered in terms of increasing immune cost, i.e., immune pathology: avoidance has a very low cost, resistance a very high cost, and tolerance lies somewhere in between (13). In addition to the cost of damage from an immune response, however, there is also an associated "energetic cost" which describes the amount of energy required to deploy a given strategy. Ordering the strategies by energetic cost indicates the same order as that of immune cost-disease avoidance very low (primarily behavioral, little to no regulation), disease resistance very high (58) (massive, highly regulated cell mobilizations across the body), and disease tolerance in the middle (some regulatory maintenance to avoid resistance and tissue healing). Both types of costs, immune and energetic, are particularly important to consider when discussing infections in neonates, as newborns are (a) particularly sensitive and prone to immunopathology (24) and (b) in the midst of a rapid growth and development phase which demands a high energy input to be maintained (59), i.e., neonates are unable to "pay" the costs of a full resistance response (Figure 2). Avoidance has failed by definition when discussing an already established active infection, which leaves disease tolerance as the primary defense strategy for newborns to cope with an invasive agent. This comprehensive, holistic point of view takes into account aspects of immunity (i.e., energy balance) which fall beyond the narrowly defined immune system and is best captured with the phrase "host fitness cost." The concept of host fitness cost helps better explain some seemingly paradoxical observations in neonatal immunity and can inform interventions moving forward.

\section{THE ROLE OF THE MICROBIOME IN NEONATAL HOST DEFENSE}

In the last decade, a vast body of research has emerged, implicating the microbiome as a critical mediator of neonatal immune development $(2,6,28,60-62)$. Dysbiosis during the neonatal period has been associated with necrotizing enterocolitis, and both early- and late-onset sepsis $(60,63-65)$. Given the potential 


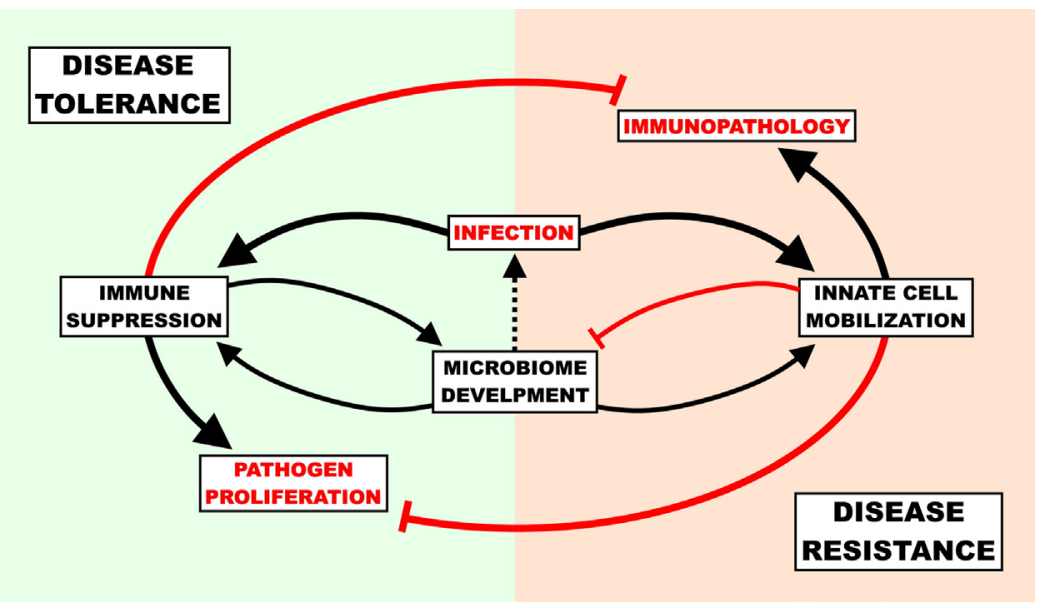

FIGURE 1 | The cost of host-defense strategies in newborn infection. The immune response must be suppressed to a degree in order to allow healthy commensal colonization of the gut, though unchecked suppression can result in gut "leakiness" and lead to infection. Upon infection, newborns must balance the potential self-inflicted harm associated with the pro-inflammatory/antimicrobial response (immunopathology) with the dangers of unencumbered pathogen proliferation and ensuing virulence. A disease tolerance strategy reduces immunopathology and supports microbiome development at the cost of pathogen load, while a disease resistance strategy reduces pathogen load at the cost of microbiome development and immunopathology.

A Energy cost: Health

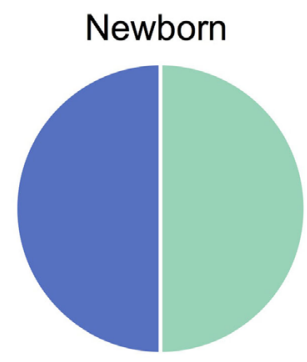

Adult

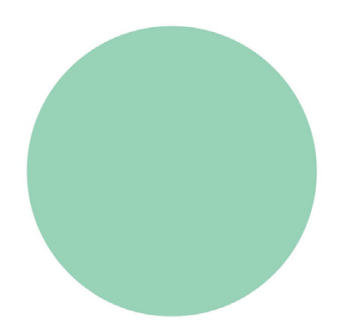

Growth \& development

Homeostasis

B Energy cost: Infectious Disease
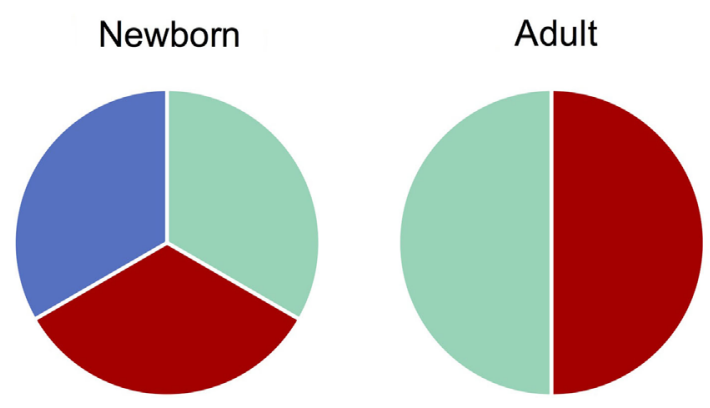

- Growth \& development

- Disease resistance

Disease tolerance

FIGURE 2 | Difference in energy demands of the newborn and adult as it relates to infectious disease. Newborns must devote a large amount of energy toward growth and development which adults are able to spend on maintaining homeostasis. When healthy (A), these differences in energetic demands may not be important, though when fighting infectious disease (B), the newborn is unable to expend the resources required to employ a strategy of disease resistance and must therefore rely more heavily on disease tolerance.

costs of impaired microbiome development, the neonatal immune system seems to have developed specific mechanisms to ensure "safe colonization" of the interphase between external and internal environments. Some of these mechanisms are reviewed below. The active immunosuppressive portion of the neonatal immune response may not only serve to minimize the damage associated 
with immunopathology but also ensure that the neonatal gut can be colonized with a large and diverse array of commensal bacteria. The interplay and dependence on commensal bacteria begins immediately with colonization, as transcriptomic analysis of germ-free mice exposed to common commensal bacteria showed the most prominent changes in genes associated with toll-like receptor (TLR) and type 1 interferon (IFN1) signaling pathways, which, as evidenced by the aforementioned importance of the TRIF-dependent signaling, are crucial in early life (66).

Newborns heavily depend on avoidance to prevent infection. In the context of microbial colonization, they rely on their mothers to introduce them to the right organisms and promote their growth, meanwhile avoiding unwanted colonizers from outcompeting the beneficial ones. While maternal influence on prepartum colonization is still being debated (67), postpartum colonization has been shown to be largely derived from the vagina during birth (68). In newborns sampled during the first week of life alongside their mothers, the majority of taxa detected in their stools were also detected in stool samples taken from their mothers at the same time (69). While newborns are colonized by bacterial families such as Bacteroides and Clostridia acquired from their mothers, the composition of their microbiota is still different from their mothers with Escherichia/Shigella, Bifidobacterium, Streptococcus, and Enterococcus occupying roughly half the space of the entire intestinal microbiome in newborns but only about $10 \%$ in their mothers [the exception is Bacteroides, which feature prominently in both (69)]. While life will eventually expose an individual to a multitude of different foods and diverse microbial environments, newborns subsist solely on breast milk and have no environmental exposure in their control. Thus, microbes found in human milk or other maternal sources that utilize human milk oligosaccharides dominate initial colonization $(70,71)$ and in turn provide resistance to colonization by potential pathogens (72) and other forms of immune support (28). Consequences of less-controlled exposure are suggested by the detriment of deviation of exclusive breastfeeding practices; excusive breastfeeding in low- and middle-income countries (and perhaps high income) is associated with a substantial reduction in newborn disease and mortality (18).

Avoiding inflammatory or deleterious responses to commensal microbes is important throughout the life span of colonized hosts. Strategies put in place by the newborn are appropriate for the environmental pressures and physiological requirements of this unique early-life period. Adults depend on a thick mucous layer packed with antimicrobial peptides and dimerized IgA alongside trained innate and adaptive mucosal responses to prevent microbial translocation into host tissues (73). Since the development of these defenses first requires stimulation by the microbiota, newborns must employ a different repertoire of tolerance strategies prior to the introduction of solid food. For example, newborn intestinal epithelial cells (IECs) produce a micro-RNA molecule that targets IRAK-1, a necessary signal transducer of inflammatory TLR signaling for degradation and thus reduces inflammation caused by commensal stimulation of intestinal TLRs (74). However, this mechanism requires continuous TLR4 stimulation for its maintenance and is absent in pups delivered by $\mathrm{C}$-section. Also, murine IECs produce antimicrobial peptide
CRAMP only prior to Paneth cell development and show some efficacy against Listeria infection (75). In fact, weaning appears to be a massive transitory period for IEC regulation. Transcriptional regulator Blimp1 is active in the newborn intestine and ceases to be expressed upon weaning; its deletion results in an adult-like intestinal architecture at birth and with it a substantial earlylife mortality in animal models (76). A more comprehensive evaluation of intestinal transcriptional regulation showed a more global postweaning shift in rodents with an increase in IL-1/TLR signaling post weaning that was lost in MyD88/TRIF ${ }^{-/}$mice, showing that the intestinal immune environment is very sensitive to changes in early-life transitions (77) and is likely guided by the changing microbial and nutritional environment.

Newborn colonizers also play an instrumental role in preventing immune hyperresponsiveness within and outside the mucosal immune system. Widely studied commensal Bacteroides fragilis promotes an anti-inflammatory environment by inhibiting the recruitment of invariant NKT cells to the gut and lung mucosa, leaving mice less susceptible to inflammatory disease later in life (78). dsRNA from lactic acid bacteria (LAB) preferentially promotes IFN- $\beta$ expression in mucosal dendritic cells to concentrations that predominantly drive their anti-inflammatory effects in adult rodents (79). Since LAB are prominent colonizers of the newborn gut, it is likely that they perform similar functions during this period-although that mechanism still needs to be investigated. An influx of highly activated regulatory $\mathrm{T}$ cells into the neonatal skin has been linked with tolerance to commensal skin bacteria, an event that was not replicated when the same experiment was performed in adult animals. Selective inhibition of these specific Tregs completely prevented tolerance to commensal bacteria colonization later in life (80).

There is evidence in adult animal models that microbiomederived products can reduce disease pathology, i.e., increase disease tolerance. Recently, the clostridia-derived metabolite desaminotyrosine (DAT) was shown to promote type 1 IFN signaling in lung dendritic cells, resulting in a less damaging response to influenza challenge and an increased animal survival, while the viral burden in DAT-treated mice remained unchanged (81). Newborns are thought to be at risk for "inside-out" infections, where the pathogen escapes mucosal compartments, supported by the identification of the same strain of bacteria from septic newborns in their feces (82). The microbiome is not only instrumental in excluding potential pathogens, but by promoting an anti-inflammatory environment, it likely also plays a role in reducing the potential harm from responses to inflammatory microbes. For example, newborn mice given probiotic strains of Lactobacillus were rescued from death caused by Citrobacter rodentium infection (a mouse model of enteropathogenic E. coli) via a mechanism involving the recruitment of Tregs to the colon (83). A second group was administered L. acidophilus alongside a prebiotic to newborn mice prior to challenge with $C$. rodentium in young adulthood, a finding that treated mice had an enhanced IL-10 and a diminished NF-kB response to infection, in addition to a faster recovery from disease (84).

Taken together, host-commensal bacteria crosstalk in newborns is highly dependent on maternal care for both original inoculation and continued support through breastfeeding and 
controlled environmental exposure. This complete dependence is unique to the newborn period, highlighted by both the devastating consequences of suboptimal breastfeeding practices and the reworking of intestinal and microbial architecture once solid foods are introduced. During this time, commensals selected to thrive in newborns promote disease tolerance by boosting anti-inflammatory immune responses and disease avoidance by excluding potentially pathogenic organisms from coming into contact with the epithelium, thus preventing infection of the mucosal and systemic sites.

\section{TRANSLATIONAL IMPACT}

Successful immune defense relies on a balance between disease resistance and disease tolerance strategies to bring the host back to homeostasis. The ideal intervention is one that would hasten restoration of homeostasis or enable the system to deal with an extreme imbalance in either direction for longer periods. A promising approach that fits this requirement is to work in the realm of innate immune memory or trained immunity-the concept that an initial infection or an exposure to a pathogen can provoke an enhanced innate immune response when the organism is re-exposed or exposed to a different pathogen (85) Unlike many traditional interventions, prophylactic or treatment approaches reliant on trained immunity are not dependent on shifting the response only toward resistance. Numerous examples of successful interventions reliant on innate immune memory have been described in animal models and human clinical trials (85). Various TLR agonists have, for example, shown to protect nonspecifically against mortality from a polymicrobial challenge $24 \mathrm{~h}$ later in neonatal mice (41). A similar model used a Listeria monocytogenes challenge and found TLR agonists to be protective as well. Cord blood monocytes stimulated with endotoxin showed an enhanced activity both 7 and 14 days later. Moreover, a retrospective analysis revealed correlation between histological chorioamnionitis (a condition prompting an inflammatory response) exposure and a reduction in late-onset neonatal sepsis (85). Most impressively, probiotics in newborns have been shown to be very powerful in reducing both necrotizing enterocolitis (86), a devastating disease characterized by colonization with proteobacteria and excessive inflammation (87), and most recently, sepsis and respiratory disease when administered within days of birth (88). Finally, certain live vaccines (particularly Bacille Calmette-Guérin) have been shown to reduce all-cause neonatal mortality, presumably through

\section{REFERENCES}

1. WHO. Causes of Child Mortality. Geneva: World Health Organization (WHO) (2016).

2. Wynn JL, Levy O. Role of innate host defenses in susceptibility to early-onset neonatal sepsis. Clin Perinatol (2010) 37:307-37. doi:10.1016/j.clp.2010.04.001

3. Gentile LF, Nacionales DC, Lopez MC, Vanzant E, Cuenca A, Cuenca AG, et al. Protective immunity and defects in the neonatal and elderly immune response to sepsis. J Immunol (2014) 192:3156-65. doi:10.4049/jimmunol.1301726

4. Wheeler DS, Wong HR, Zingarelli B. Pediatric sepsis-part I: 'children are not small adults!.' Open Inflamm J (2011) 4:4-15. doi:10.2174/1875041901104010004 nonspecific protection against unrelated pathogens in the first month of life (89).

\section{SUMMARY}

The paradigm that neonates are more susceptible to infectious disease than adults is well known, well documented, yet poorly understood. The high susceptibility and mortality figures have largely been attributed to "immune immaturity," a vague concept that is predicated on findings of weaker antimicrobial responses of newborns than those of adults. Here, we posit that an increased susceptibility to infection in neonates is not a result of immaturity but rather one of immunosuppressions, which is in part an active defense strategy termed disease tolerance. This is supported by the finding that neonates can survive significantly higher bacterial loads than adults during active infection. This observation is consistent across many studies, yet still is oft ignored and cannot be adequately explained by the immaturity paradigm. Employing a defense strategy of disease tolerance during infection rather than disease resistance confers some advantages but is more likely a virtue of necessity. Compared to the adult-like disease resistance strategy, disease tolerance is (a) less energetically intensive (critical during a period of rapid development), (b) less likely to incur serious damage associated with bacterial clearance (neonates are more sensitive to immunopathology than adults and seem to have a heightened innate immune response), and (c) less likely to interfere with the development of the gut microbiome (overactive resistance pathways could result in a dangerous inflammatory response and interfere with colonization). Maintaining homeostasis between disease resistance and disease tolerance is a critical outcome of fighting and preventing infection. Fortunately, interventions, which work within these constraints, have been identified and promise to finally usher in the desperately needed reduction of global newborn mortality rates.

\section{AUTHOR CONTRIBUTIONS}

$\mathrm{DH}, \mathrm{NA}$, and TK conceived of the presented ideas. DH wrote the manuscript with assistance from RB-O, NH, and TK. All authors provided critical feedback and editing through the writing process.

\section{FUNDING}

This research was supported by a Michael Smith Foundation for Health Research Career Investigator Award to TK.

5. Raymond SL, Stortz JA, Mira JC, Larson SD, Wynn JL, Moldawer LL. Immunological defects in neonatal sepsis and potential therapeutic approaches. Front Pediatr (2017) 5:14. doi:10.3389/fped.2017.00014

6. Yoon HS. Neonatal innate immunity and toll-like receptor. Korean J Pediatr (2010) 53:985-8. doi:10.3345/kjp.2010.53.12.985

7. Kai-Larsen Y, Gudmundsson GH, Agerberth B. A review of the innate immune defence of the human foetus and newborn, with the emphasis on antimicrobial peptides. Acta Paediatr (2014) 103:1000-8. doi:10.1111/apa.12700

8. Wynn JL, Neu J, Moldawer LL, Levy O. Potential of immunomodulatory agents for prevention and treatment of neonatal sepsis. J Perinatol (2009) 29:79-88. doi:10.1038/jp.2008.132 
9. Marshall JC. Why have clinical trials in sepsis failed? Trends Mol Med (2014) 20:195-203. doi:10.1016/j.molmed.2014.01.007

10. Maddux $A B$, Douglas IS. Is the developmentally immature immune response in paediatric sepsis a recapitulation of immune tolerance? Immunology (2015) 145:1-10. doi:10.1111/imm.12454

11. Brook B, Harbeson D, Ben-Othman R, Viemann D, Kollmann TR. Newborn susceptibility to infection vs. disease depends on complex in vivo interactions of host and pathogen. Semin Immunopathol (2017) 1-11(6):615-25. doi:10.1007/s00281-017-0651-z

12. Kollmann TR, Kampmann B, Mazmanian SK, Marchant A, Levy O. Protecting the newborn and young infant from infectious diseases: lessons from immune ontogeny. Immunity (2017) 46:350-63. doi:10.1016/j.immuni.2017.03.009

13. Medzhitov R, Schneider DS, Soares MP. Disease tolerance as a defense strategy. Science (2012) 335:936-42. doi:10.1126/science.1214935

14. Li YP, Yu SL, Huang ZJ, Huang J, Pan J, Feng X, et al. An impaired inflammatory cytokine response to Gram-negative LPS in human neonates is associated with the defective TLR-mediated signaling pathway. J Clin Immunol (2015) 35:218-26. doi:10.1007/s10875-015-0128-6

15. Cuenca AG, Wynn JL, Moldawer LL, Levy O. Role of innate immunity in neonatal infection. Am J Perinatol (2013) 30:105-12. doi:10.1055/s-00321333412

16. Alford TJ, Roberts WE, Hartsfield JK, Eckert GJ, Snyder RJ. Differences between adult and pediatric septic shock. Minerva Med (2011) 77:1-7.

17. Fujiwara T, Kobayashi T, Takaya J, Taniuchi S, Kobayashi Y. Plasma effects on phagocytic activity and hydrogen peroxide production by polymorphonuclear leukocytes in neonates. Clin Immunol Immunopathol (1997) 85:67-72. doi:10.1006/clin.1997.4403

18. Victora CG, Bahl R, Barros AJ, França GV, Horton S, Krasevec J, et al. Breastfeeding in the 21st century: epidemiology, mechanisms, and lifelong effect. Lancet (2016) 387:475-90. doi:10.1016/S0140-6736(15)01024-7

19. Bone RC, Balk RA, Cerra FB, Dellinger RP, Fein AM, Knaus WA, et al. Definitions for sepsis and organ failure and guidelines for the use of innovative therapies in sepsis. The ACCP/SCCM Consensus Conference Committee. American College of Chest Physicians/Society of Critical Care Medicine. Chest (1992) 101:1644-55. doi:10.1378/chest.101.6.1644

20. Seemann S, Zohles F, Lupp A. Comprehensive comparison of three different animal models for systemic inflammation. J Biomed Sci (2017) 24:60. doi:10.1186/s12929-017-0370-8

21. Willenberg I, Rund K, Rong S, Shushakova N, Gueler F, Schebb NH. Characterization of changes in plasma and tissue oxylipin levels in LPS and CLP induced murine sepsis. Inflamm Res (2016) 65:133-42. doi:10.1007/ s00011-015-0897-7

22. Peñaloza HF, Nieto PA, Muñoz-Durango N, Salazar-Echegarai FJ, Torres J, Parga MJ, et al. Interleukin-10 plays a key role in the modulation of neutrophils recruitment and lung inflammation during infection by Streptococcus pneumoniae. Immunology (2015) 146:100-12. doi:10.1111/imm.12486

23. Tang-Feldman YJ, Lochhead GR, Lochhead SR, Yu C, Pomeroy C. Interleukin-10 repletion suppresses pro-inflammatory cytokines and decreases liver pathology without altering viral replication in murine cytomegalovirus (MCMV)-infected IL-10 knockout mice. Inflamm Res (2010) 60:233-43. doi:10.1007/s00011-010-0259-4

24. Zhao J, Kim KD, Yang X, Auh S, Fu YX, Tang H. Hyper innate responses in neonates lead to increased morbidity and mortality after infection. Proc Natl Acad Sci U S A (2008) 105:7528-33. doi:10.1073/pnas.0800152105

25. Elgin TG, Kern SL, McElroy SJ. Development of the neonatal intestinal microbiome and its association with necrotizing enterocolitis. Clin Ther (2016) 38:706-15. doi:10.1016/j.clinthera.2016.01.005

26. Wynn JL, Scumpia PO, Delano MJ, O’Malley KA, Ungaro R, Abouhamze A, et al. Increased mortality and altered immunity in neonatal sepsis produced by generalized peritonitis. Shock (2007) 1(6):675-83. doi:10.1097/ SHK.0b013e3180556d09

27. Simms EL, Triplett J. Costs and benefits of plant responses to disease: resistance and tolerance. Evolution (1994) 48:1973-85. doi:10.1111/j.1558-5646.1994. tb02227.x

28. Gritz EC, Bhandari V. The human neonatal gut microbiome: a brief review. Front Pediatr (2015) 3:17. doi:10.3389/fped.2015.00017

29. Cantey JB, Wozniak PS, Sánchez PJ. Prospective surveillance of antibiotic use in the neonatal intensive care unit. Pediatr Infect Dis J (2015) 34:267-72. doi:10.1097/INF.0000000000000542
30. Hornik CP, Benjamin DK, Becker KC, Benjamin DK Jr, Li J, Clark RH, et al. Use of the complete blood cell count in early-onset neonatal sepsis. Pediatr Infect Dis J (2012) 31:799-802. doi:10.1097/INF.0b013e318256905c

31. Yagupsky P, Nolte FS. Quantitative aspects of septicemia. Clin Microbiol Rev (1990) 3:269-79. doi:10.1128/CMR.3.3.269

32. Werner AS, Cobbs CG, Kaye D, Hook EW. Studies on the bacteremia of bacterial endocarditis. JAMA (1967) 202:199-203. doi:10.1001/jama.1967. 03130160073013

33. Kreger BE, Craven DE, Carling PC, McCabe WR. Gram-negative bacteremia. III. Reassessment of etiology, epidemiology and ecology in 612 patients. Am J Med (1980) 68:332-43. doi:10.1016/0002-9343(80)90101-1

34. Henry NK, McLimans CA, Wright AJ, Thompson RL, Wilson WR, Washington JA II. Microbiological and clinical evaluation of the isolator lysis-centrifugation blood culture tube. J Clin Microbiol (1983) 17:864-9.

35. Dietzman DE, Fischer GW, Schoenknecht FD. Neonatal Escherichia coli septicemia-bacterial counts in blood. J Pediatr (1974) 85:128-30. doi:10.1016/ S0022-3476(74)80308-2

36. Zhang Q, Coveney AP, Yu S, Liu JH, Li Y, Blankson S, et al. Inefficient antimicrobial functions of innate phagocytes render infant mice more susceptible to bacterial infection. Eur J Immunol (2013) 43:1322-32. doi:10.1002/eji.201243077

37. HALL WH, GOLD D. Shock associated with bacteremia. AMA Arch Intern Med (1955) 96:403. doi:10.1001/archinte.1955.00250140125014

38. WEIL MH, SPINK WW. The shock syndrome associated with bacteremia due to Gram-negative bacilli. Arch Intern Med (1958) 101:184. doi:10.1001/ archinte.1958.00260140016004

39. Fitzpatrick EA, You D, Shrestha B, Siefker D, Patel VS, Yadav N, et al. A neonatal murine model of MRSA pneumonia. PLoS One (2017) 12:e0169273. doi:10.1371/journal.pone. 0169273

40. Filias A, Theodorou GL, Mouzopoulou S, Varvarigou AA, Mantagos S, Karakantza M. Phagocytic ability of neutrophils and monocytes in neonates. BMC Pediatr (2011) 11:29. doi:10.1186/1471-2431-11-29

41. Wynn JL, Scumpia PO, Winfield RD, Delano MJ, Kelly-Scumpia K, Barker T, et al. Defective innate immunity predisposes murine neonates to poor sepsis outcome but is reversed by TLR agonists. Blood (2008) 112:1750-8. doi:10.1182/blood-2008-01-130500

42. ElahiS, ErteltJM, KinderJM, JiangTT,ZhangX, Xin L, etal. Immunosuppressive CD71+ erythroid cells compromise neonatal host defence against infection. Nature (2013) 504:158-62. doi:10.1038/nature12675

43. Hallwirth U, Pomberger G, Pollak A, Roth E, Spittler A. Monocyte switch in neonates: high phagocytic capacity and low HLA-DR expression in VLBWI are inverted during gestational aging. Pediatr Allergy Immunol (2004) 15:513-6. doi:10.1111/j.1399-3038.2004.00168.x

44. Silveira-Lessa AL, Quinello C, Lima L, Redondo ACC, Ceccon MEJR, CarneiroSampaio M, et al. TLR expression, phagocytosis and oxidative burst in healthy and septic newborns in response to Gram-negative and Gram-positive rods. Hum Immunol (2016) 77:972-80. doi:10.1016/j.humimm.2016.07.230

45. Carr R. Neutrophil production and function in newborn infants. Br J Haematol (2000) 110:18-28. doi:10.1046/j.1365-2141.2000.01992.x

46. Kretschmer RR, Stewardson PB, Cynthia K, Gotoff SP, Gotoff SP. Chemotactic and bactericidal capacities of human newborn monocytes. J Immunol (1976) 117:1303-7.

47. Gille Ch, Leiber A, Mundle I, Spring B, Abele H, Spellerberg B, et al. Phagocytosis and postphagocytic reaction of cord blood and adult blood monocyte after infection with green fluorescent protein-labeled Escherichia coli and group B Streptococci. Cytometry B Clin Cytom (2009) 76B:271-84. doi:10.1002/cyto.b.20474

48. Ashare A, Powers LS, Butler NS, Doerschug KC, Monick MM, Hunninghake GW. Anti-inflammatory response is associated with mortality and severity of infection in sepsis. Am J Physiol Lung Cell Mol Physiol (2004) 288:L633-40. doi:10.1152/ajplung.00231.2004

49. Ward NS, Casserly B, Ayala A. The compensatory anti-inflammatory response syndrome (CARS) in critically ill patients. Clin Chest Med (2008) 29:617-25,viii. doi:10.1016/j.ccm.2008.06.010

50. Evans I, Jones C. HSV induces an early primary Th1 CD4 T? Cell response in neonatal mice, but reduced CTL activity at the time of the peak adult response. Eur J Immunol (2005) 35:1454-62. doi:10.1002/eji.200425333

51. Echeverry A, Saijo S, Schesser K, Adkins B. Yersinia enterocolitica promotes robust mucosal inflammatory T-cell immunity in murine neonates. Infect Immun (2010) 78:3595-608. doi:10.1128/IAI.01272-09 
52. Kronforst KD, Mancuso CJ, Pettengill M, Ninkovic J, Power-Coombs MR, Stevens C, et al. A neonatal model of intravenous Staphylococcus epidermidis infection in mice. PLoS One (2012) 7:e43897. doi:10.1371/journal. pone.0043897

53. Wynn JL, Wilson CS, Hawiger J, Scumpia PO, Marshall AF, Liu JH, et al. Targeting IL-17A attenuates neonatal sepsis mortality induced by IL-18. Proc Natl Acad Sci U S A (2016) 113:E2627-35. doi:10.1073/pnas.1515793113

54. Aziz M, Jacob A, Yang W, Matsuda A, Wang P. Current trends in inflammatory and immunomodulatory mediators in sepsis. J Leukoc Biol (2012) 93:329-42. doi:10.1189/jlb.0912437

55. Cuenca AG, Joiner DN, Gentile LF, Cuenca AL, Wynn JL, Kelly-Scumpia KM, et al. TRIF-dependent innate immune activation is critical for survival to neonatal Gram-negative sepsis. J Immunol (2015) 194:1169-77. doi:10.4049/ jimmunol.1302676

56. Ulas T, Pirr S, Fehlhaber B, Bickes MS, Loof TG, Vogl T, et al. S100-alarmininduced innate immune programming protects newborn infants from sepsis. Nat Immunol (2017) 18:622-32. doi:10.1038/ni.3745

57. Torrazza RM, Neu J. The developing intestinal microbiome and its relationship to health and disease in the neonate. J Perinatol (2011) 31:S29-34. doi:10.1038/jp.2010.172

58. Iwasaki A, Medzhitov R. Control of adaptive immunity by the innate immune system. Nat Immunol (2015) 16:343-53. doi:10.1038/ni.3123

59. Falkner F, Tanner JM. Human growth: a comprehensive treatise. In: Falkner F, Tanner JM, editors. Developmental Biology Prenatal Growth Postnatal Growth Neurobiology Methodology Ecological, Genetic, and Nutritional Effects on Growth. (Vol. 2), New York: Springer Science (1986). p. 101-17.

60. Deshmukh HS, Liu Y, Menkiti OR, Mei J, Dai N, O’Leary CE, et al. The microbiota regulates neutrophil homeostasis and host resistance to Escherichia coli K1 sepsis in neonatal mice. Nat Med (2014) 20:524-30. doi:10.1038/nm.3542

61. Cho I, Blaser MJ. The human microbiome: at the interface of health and disease. Nat Rev Genet (2012) 13:260. doi:10.1038/nrg3182

62. Gensollen T, Iyer SS, Kasper DL, Blumberg RS. How colonization by microbiota in early life shapes the immune system. Science (2016) 352:539-44. doi:10.1126/science.aad9378

63. Afsharpaiman S, Torkaman M, Saburi A, Farzaampur A, Amirsalari S, Kavehmanesh Z. Trends in incidence of neonatal sepsis and antibiotic susceptibility of causative agents in two neonatal intensive care units in Tehran, I.R Iran. J Clin Neonatol (2012) 1:124-30. doi:10.4103/2249-4847.101692

64. Alm B, Erdes L, Möllborg P, Pettersson R, Norvenius SG, Aberg N, et al. Neonatal antibiotic treatment is a risk factor for early wheezing. Pediatrics (2008) 121:697-702. doi:10.1542/peds.2007-1232

65. Vael C, Desager K. The importance of the development of the intestinal microbiota in infancy. Curr Opin Pediatr (2009) 21:794-800. doi:10.1097/ MOP.0b013e328332351b

66. Yamamoto M, Yamaguchi R, Munakata K, Takashima K, Nishiyama M, Hioki K, et al. A microarray analysis of gnotobiotic mice indicating that microbial exposure during the neonatal period plays an essential role in immune system development. BMC Genomics (2012) 13:335. doi:10.1186/1471-2164-13-335

67. Perez-Muñoz ME, Arrieta M-C, Ramer-Tait AE, Walter J. A critical assessment of the 'sterile womb' and 'in utero colonization' hypotheses: implications for research on the pioneer infant microbiome. Microbiome (2017) 5:48. doi:10.1186/s40168-017-0268-4

68. Dominguez-Bello MG, Costello EK, Contreras M, Magris M, Hidalgo G, Fierer N, et al. Delivery mode shapes the acquisition and structure of the initial microbiota across multiple body habitats in newborns. Proc Natl Acad Sci US A (2010) 107:11971-5. doi:10.1073/pnas.1002601107

69. Bäckhed F, Roswall J, Peng Y, Feng Q, Jia H, Kovatcheva-Datchary P, et al. Dynamics and stabilization of the human gut microbiome during the first year of life. Cell Host Microbe (2015) 17:690-703. doi:10.1016/j.chom.2015.04.004

70. Marcobal A, Sonnenburg JL. Human milk oligosaccharide consumption by intestinal microbiota. Clin Microbiol Infect (2012) 18:12-5. doi:10.1111/ j.1469-0691.2012.03863.x

71. Jost T, Lacroix C, Braegger C, Chassard C. Impact of human milk bacteria and oligosaccharides on neonatal gut microbiota establishment and gut health. Nutr Rev (2015) 73:426-37. doi:10.1093/nutrit/nuu016

72. Heikkila MP, Saris PEJ. Inhibition of Staphylococcus aureus by the commensal bacteria of human milk. J Appl Microbiol (2003) 95:471-8. doi:10.1046/ j.1365-2672.2003.02002.x
73. Duerr CU, Hornef MW. The mammalian intestinal epithelium as integral player in the establishment and maintenance of host-microbial homeostasis. Semin Immunol (2012) 24:25-35. doi:10.1016/j.smim.2011.11.002

74. Chassin C, Kocur M, Pott J, Duerr CU, Gütle D, Lotz M, et al. miR-146a mediates protective innate immune tolerance in the neonate intestine. Cell Host Microbe (2010) 8:358-68. doi:10.1016/j.chom.2010.09.005

75. Ménard S, Förster V, Lotz M, Gütle D, Duerr CU, Gallo RL, et al. Developmental switch of intestinal antimicrobial peptide expression. J Exp Med (2008) 205:183-93. doi:10.1084/jem.20071022

76. Muncan V, Heijmans J, Krasinski SD, Büller NV, Wildenberg ME, Meisner S, et al. Blimp1 regulates the transition of neonatal to adult intestinal epithelium. Nat Commun (2011) 2:452. doi:10.1038/ncomms1463

77. Rakoff-Nahoum S, Kong Y, Kleinstein SH, Subramanian S, Ahern PP, Gordon JI, et al. Analysis of gene-environment interactions in postnatal development of the mammalian intestine. Proc Natl Acad Sci U S A (2015) 112:1929-36. doi:10.1073/pnas.1424886112

78. An D, Oh SF, Olszak T, Neves JF, Avci FY, Erturk-Hasdemir D, et al. Sphingolipids from a symbiotic microbe regulate homeostasis of host intestinal natural killer T cells. Cell (2014) 156:123-33. doi:10.1016/j.cell.2013.11.042

79. Kawashima T, Kosaka A, Yan H, Guo Z, Uchiyama R, Fukui R, et al. Double-stranded RNA of intestinal commensal but not pathogenic bacteria triggers production of protective interferon- $\beta$. Immunity (2013) 38:1187-97. doi:10.1016/j.immuni.2013.02.024

80. Scharschmidt TC, Vasquez KS, Truong HA, Gearty SV, Pauli ML, Nosbaum A, et al. A wave of regulatory $\mathrm{T}$ cells into neonatal skin mediates tolerance to commensal microbes. Immunity (2015) 43:1011-21. doi:10.1016/j.immuni. 2015.10.016

81. Steed AL, Christophi GP, Kaiko GE, Sun L, Goodwin VM, Jain U, et al. The microbial metabolite desaminotyrosine protects from influenza through type I interferon. Science (2017) 502:498-502. doi:10.1126/science.aam5336

82. Das P, Singh AK, Pal T, Dasgupta S, Ramamurthy T, Basu S. Colonization of the gut with Gram-negative bacilli, its association with neonatal sepsis and its clinical relevance in a developing country. J Med Microbiol (2011) 60:1651-60. doi:10.1099/jmm.0.033803-0

83. Gareau MG, Sherman PM, Walker WA. Probiotics and the gut microbiota in intestinal health and disease. Nat Rev Gastroenterol Hepatol (2010) 7:503-14. doi:10.1038/nrgastro.2010.117

84. Foye OT, Huang I-F, Chiou CC, Walker WA, Shi HN. Early administration of probiotic Lactobacillus acidophilus and/or prebiotic inulin attenuates pathogen-mediated intestinal inflammation and Smad 7 cell signaling. FEMS Immunol Med Microbiol (2012) 65:467-80. doi:10.1111/j.1574-695X.2012.00978.x

85. Levy O, Wynn JL. A prime time for trained immunity: innate immune memory in newborns and infants. Neonatology (2014) 105:136-41. doi:10.1159/ 000356035

86. AlFaleh K, Anabrees J, Bassler D, Al-Kharfi T. Probiotics for prevention of necrotizing enterocolitis in preterm infants. In: AlFaleh $\mathrm{K}$, editor. Cochrane Database of Systematic Reviews. Chichester, UK: John Wiley \& Sons, Ltd (2014).

87. Neu J, Walker WA. Necrotizing enterocolitis. N Engl J Med (2011) 364:255-64. doi:10.1056/NEJMra1005408

88. Panigrahi P, Parida S, Nanda NC, Satpathy R, Pradhan L, Chandel DS, et al. A randomized synbiotic trial to prevent sepsis among infants in rural India. Nature (2017) 548:407-12. doi:10.1038/nature23480

89. Goodridge HS, Ahmed SS, Curtis N, Kollmann TR, Levy O, Netea MG, et al. Harnessing the beneficial heterologous effects of vaccination. Nat Rev Immunol (2016) 16:392-400. doi:10.1038/nri.2016.43

Conflict of Interest Statement: The authors declare that the research was conducted in the absence of any commercial or financial relationships that could be construed as a potential conflict of interest.

Copyright (c) 2018 Harbeson, Ben-Othman, Amenyogbe and Kollmann. This is an open-access article distributed under the terms of the Creative Commons Attribution License (CC BY). The use, distribution or reproduction in other forums is permitted, provided the original author(s) and the copyright owner are credited and that the original publication in this journal is cited, in accordance with accepted academic practice. No use, distribution or reproduction is permitted which does not comply with these terms. 